\title{
rANOMALY: AmplicoN wOrkflow for Microbial community
}

\section{AnaLYsis [version 1; peer review: 2 approved]}

\section{Sebastien Theil ${ }^{\star}$, Etienne Rifa*}

INRAE, F-15000, Aurillac, France

${ }^{*}$ Equal contributors

V1 First published: 07 Jan 2021, 10:7

https://doi.org/10.12688/f1000research.27268.1

Latest published: 07 Jan 2021, 10:7

https://doi.org/10.12688/f1000research.27268.1

\section{Abstract}

Bioinformatic tools for marker gene sequencing data analysis are continuously and rapidly evolving, thus integrating most recent techniques and tools is challenging. We present an R package for data analysis of $16 \mathrm{~S}$ and ITS amplicons based sequencing. This workflow is based on several $R$ functions and performs automatic treatments from fastq sequence files to diversity and differential analysis with statistical validation. The main purpose of this package is to automate bioinformatic analysis, ensure reproducibility between projects, and to be flexible enough to quickly integrate new bioinformatic tools or statistical methods. rANOMALY is an easy to install and customizable $R$ package, that uses amplicon sequence variants (ASV) level for microbial community characterization. It integrates all assets of the latest bioinformatics methods, such as better sequence tracking, decontamination from control samples, use of multiple reference databases for taxonomic annotation, all main ecological analysis for which we propose advanced statistical tests, and a cross-validated differential analysis by four different methods. Our package produces ready to publish figures, and all of its outputs are made to be integrated in Rmarkdown code to produce automated reports.

\section{Keywords}

16S, ITS, amplicon sequencing, metagenomics, microbial community, $\mathrm{R}$ package

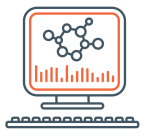

This article is included in the Bioinformatics gateway.

\section{Open Peer Review \\ Approval Status \\ 1 2 \\ version 1 \\ 07 Jan 2021
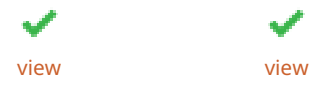

1. Florent Murat, Heidelberg University, Heidelberg, Germany

\section{Simon Roux, Department of Energy Joint}

Genome Institute, Berkeley, USA

Any reports and responses or comments on the article can be found at the end of the article. 
Corresponding authors: Sebastien Theil (sebastien.theil@inrae.fr), Etienne Rifa (etienne.rifa@inrae.fr)

Author roles: Theil S: Conceptualization, Methodology, Software, Validation, Writing - Original Draft Preparation, Writing - Review \& Editing; Rifa E: Conceptualization, Methodology, Software, Validation, Writing - Original Draft Preparation, Writing - Review \& Editing Competing interests: No competing interests were disclosed.

Grant information: The author(s) declared that no grants were involved in supporting this work.

Copyright: ๑ 2021 Theil S and Rifa E. This is an open access article distributed under the terms of the Creative Commons Attribution License, which permits unrestricted use, distribution, and reproduction in any medium, provided the original work is properly cited.

How to cite this article: Theil S and Rifa E. rANOMALY: AmplicoN wOrkflow for Microbial community AnaLYsis [version 1; peer review: 2 approved] F1000Research 2021, 10:7 https://doi.org/10.12688/f1000research.27268.1

First published: 07 Jan 2021, 10:7 https://doi.org/10.12688/f1000research.27268.1 


\section{Introduction}

Studies of microbial communities tends to become a daily routine analysis for lots of laboratories and the main method to explore microbial diversity is metabarcoding, which is an amplicon targeted sequencing method (16S for bacteria and ITS for fungi). Metabarcoding generates a large amount of data and a lot of applications already exist for their processing (FROGS ${ }^{1}$, qiime $^{2}$ ). Methods and software are continuously evolving and the main challenge for bioinformaticians is to implement the most recent and effective ones in their analysis. Here we present rANOMALY, a scalable and lightweight $\mathrm{R}$ package which is able to handle every step of a metabarcoding analysis, from read cleaning, contaminant filtering, taxonomic assignment, to advanced statistical analysis. rANOMALY is fully implemented in R language in which each step correspond to one function, allowing to easy implementation of new features or tools while being easy to use and maintain. The package allows the workflow to be executed on any R environment. rANOMALY only needs a CSV table describing the metadata for each sample, and a folder containing the corresponding fastq files as input. It can produce high quality figures for Rmarkdown reports along with statistical tests ready for publication.

The workflow is illustrated in Figure 1.

\section{Implementation}

rANOMALY is an R package depending on other CRAN, Bioconductor, and git R packages. It is easy to install via devtools::install_git function.

\section{Raw sequence processing}

Samples must have been previously demultiplexed into one file per sample with the file name following this syntax: \{sampleid\}_R[12].fastq. The denoising process is handled using the dada2 $\mathrm{R}$ package ${ }^{3}$ which produces amplicon sequence variants (ASV) as a taxonomic unit. This improves resolution of the potential presence of microbial organisms by using a prediction model to correct sequencing errors before aggregating similar sequences. rANOMALY handles processing any region of $16 \mathrm{~S}$ (V1 to V9) and ITS amplicon (ITS1, ITS2) sequences, in which an additional step of cutadapt ${ }^{4}$ removes ITS probes left in some short sequences. For $16 \mathrm{~S}$ amplicon, primers are trimmed based on the primer sequence length. ASV identifiers are sequences translated into MD5 hashes which are unique identifiers based on the DNA sequences offering the possibility to be

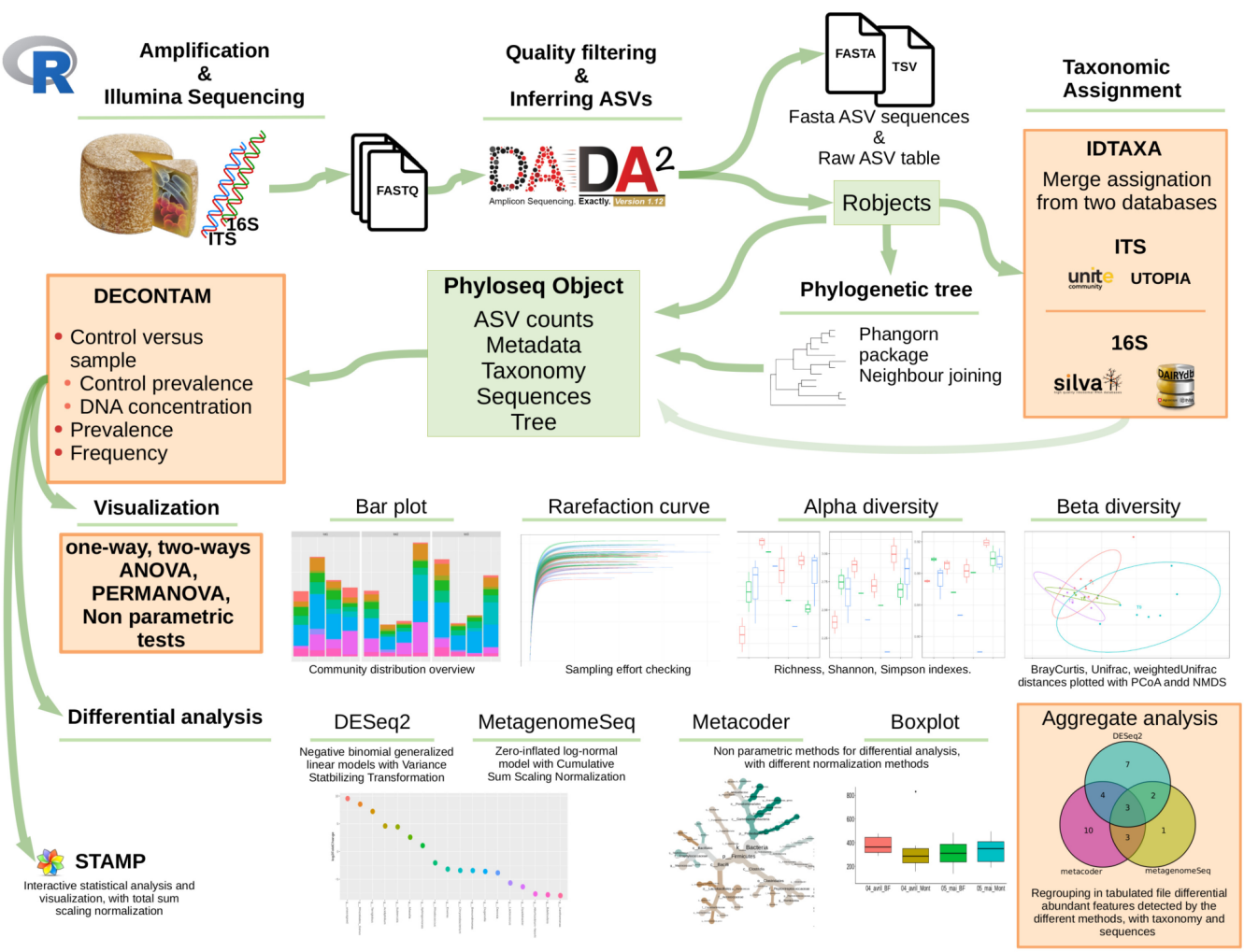

Figure 1. rANOMALY workflow. 
compared between projects. This step results in an object with representing sequences, a raw ASV counts table and a text file containing statistics from the denoising process.

\section{Taxonomic assignment}

Taxonomic assignments of ASVs are carried out by IDTAXA (part of the DECIPHER package), an algorithm based on a machine learning method ${ }^{5}$. We implemented a functionality to compute assignment with two reference databases. For example, 16S amplicon sequences can be assigned with an environment specific database $\left(\right.$ DAIRYdb $\left.^{6}, \mathrm{HITdb}^{7}, \mathrm{MIDAS}^{8}\right)$ and a general database like SILVA $^{9}$ or GreenGenes ${ }^{10}$. The assignment with the best confidence and the lowest rank is kept, thus increasing assignment depth and accuracy. As taxonomy can differ between reference databases, we implemented a taxonomy validation step in rANOMALY to unravel taxonomy inconsistency like taxa with multiple ancestors or empty ranks. As supplementary features, rANOMALY functions allow users to create their own IDTAXA formatted specific reference database. The first step consists of filling the taxonomic table empty fields with the last known rank, and checking for taxonomy incongruencies as in the assignment function. A taxid file as used with RDP classifier ${ }^{11}$ is constructed and then a last function takes as input, the corrected taxonomy table, the fasta file and the taxid file to generate the IDTAXA formatted database.

\section{Phylogenetic tree}

Phylogenetic tree is generated in three steps. First, sequences are aligned with AlignSeqs function from DECIPHER package ${ }^{12}$ by the guided-tree method. Then, distance matrix is calculated with the dist.ml function from the phangorn package. And finally, neighbour joining and pml function computes the likelihood of the phylogenetic tree.

The abundance, metadata, taxonomic table, reference sequences and phylogenetic tree are merged into a phyloseq object ${ }^{13}$.

\section{Decontamination}

ASVs have the advantage of enabling the distinction between contaminants and the real community. We have integrated the decontam package ${ }^{14}$ into the workflow. Indeed using OTU based clustering methods can agglomerate contaminant sequences with real sample sequences, the whole cluster could hence be considered as contaminant by mistake. Working with ASVs allows the use of R package decontam which will sensitively exclude contaminant ASVs. It integrates two main methods, one based on the prevalence of the contaminant in the control samples, and another one based on the DNA concentration of the samples. Moreover, our decontamination step allows users to apply various filters such as low ASVs frequency, low ASVs prevalence in real samples, and the minimum number of reads per sample.

\section{Graphical plots and statistical analyses}

Statistical analyses are key features of rANOMALY workflow. Main descriptive analyses are integrated thanks to phyloseq functionalities. In addition, we automatized graphical representations and advanced statistical tests for alpha, beta diversity and composition plots. Above all, we have included the four most up-to-date differential analyses to assess differentially abundant features.

Community composition plots, alpha and beta diversity analyses

rANOMALY allows users to explore microbial community composition with three different types of plots : classical interactive bar plot $^{15}$ of raw and relative taxa abundances, rarefaction curves to check sampling effort, and Krona interactive pie charts ${ }^{16}$.

Alpha diversity indices representing the specific richness are calculated (Richness, Simpson, Shannon...) with the vegan $\mathrm{R}$ package ${ }^{17}$. We added statistical tests such as multi-factors analysis of variance and pairwise Wilcoxon tests to assess significant differences between tested categories. We included repeated measures ANOVA to handle within-subjects variation. For example, it can be used when there are measures taken on the same individuals at different time points. This step outputs graphical representations and tables with results of statistical tests which are both saved in files and returned as list objects for markdown reports.

Beta diversity analysis allows users to estimate the community differences between two samples, it is also based on the vegan package ${ }^{17}$. rANOMALY can calculate all different distances such as the one based on ASVs abundance (BrayCurtis), rank based Jaccard indices, and phylogenetic distances as UniFrac, weighted Unifrac. Graphical representations PCoA, NMDS and more are available. This analysis can be processed at different taxonomic levels and categorical factors chosen by the user. The additional statistical test of PERMANOVA uses the distance matrix to determine if microbial communities of sample groups are significantly different 
from each other. We added a pairwise PERMANOVA ${ }^{18}$ test to confirm significant differences between specific group of samples.

\section{Differential analysis}

Differential analyses are meant to assess potential differentially abundant taxas between tested conditions chosen by users. rANOMALY wraps three methods:

- DESeq $2^{19}$ uses negative binomial generalized linear model with a variance stabilizing transformation on abundances.

- metagenomeSeq ${ }^{20}$ uses a zero-inflated log-normal model with cumulative sum scaling normalization.

- metacoder $^{21}$ applies a total sample sum normalisation and uses a non-parametric Wilcoxon Rank Sum test to compare the log ratio of mean proportions.

The use of multiple methods for differential analysis allows the user to investigate which feature can be considered as differentially abundant between conditions. rANOMALY function using DESeq2 and metagenomeSeq outputs tables and plots with significant features. Metacoder outputs heat tree plots allowing users to infer differentially abundant features at each taxonomy rank and their position on the phylogenetic tree. With basic data management, and according to the identification of all significant differentially features (i.e. ASVs, genus, species) from the three methods, we generate a single table to find significant features in one or more methods and ease the interpretation. Additional information are added to the final table, like mean relative abundance for each feature and condition. A column in which condition features are significantly more abundant, features taxonomies and related sequences are added to the aggregated table. To complete differential analysis, we have included the well recognized PLS-DA from the mixOmics package ${ }^{22}$. It is a supervised classification method allowing users to identify features discriminating the sample groups.

\section{Additional features and export}

Functions and procedures are available to help the user to generate additional figures or to export the data to third party software. For instance, shared taxa between conditions are useful to explore. We use a function that can generate Venn diagrams, or for more complex visualisation, we can produce files readable by Cytoscape $^{23}$ to produce shared taxa networks. Krona diagrams can be displayed to explore sample microbial composition, where samples can be merged by a specified factor. rANOMALY allows users to generate inputs for STAMP24, which is a graphical software that provides statistical hypothesis tests and exploratory plots for analysing taxonomic profiles.

\section{Operation}

rANOMALY requires R 3.6 .3 or upper and can be run on any operating system with common specifications (1Go disk space, 4Go RAM, multicore CPU is recommended).

\section{Use case}

For this example we are using a dataset from Fretin et al. study ${ }^{25}$ in which samples are from four different environments: cow milk, cow cheese (rind and core) and cow teat skin. This dataset and metadata are available on NCBI-SRA website: BioProject accession PRJNA421256. To ease access to this dataset, fastq files along with pre-formatted metadata are available on this repository.

\section{Install}

Up to date code is hosted by the INRAE gitlab, users can simply download and install the package in R console with following command lines.

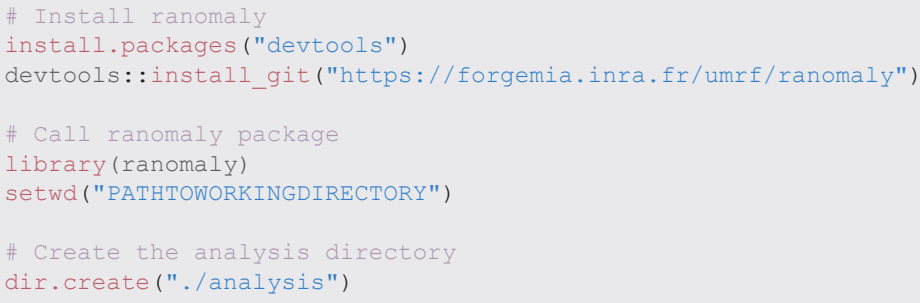




\section{Processing of raw sequences}

$\boldsymbol{A S V}$ definition with DADA2. The first step is to define ASVs thanks to the dada 2 package. In $r A N O M A L Y$, only one function is needed to compute all the different steps require from this package. Here sample names are extracted from the file name, thus be sure that files name match samples name in the metadata file.

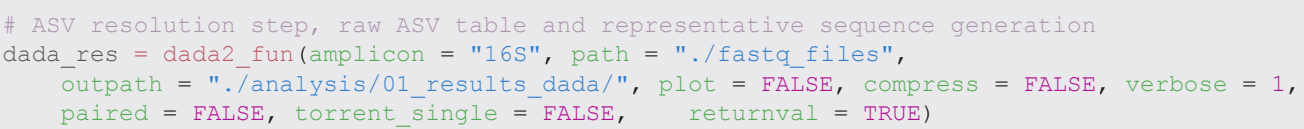

Main outputs of this function are:

- read_tracking. csv summarizes the read number after each filtering step (Table 1).

- raw_otu-table.csv the raw ASV table.

- rep-seqs. fna fasta file with all representative sequences for each ASV.

- robjects.Rdata with saved dada res list containing raw ASV table and representative sequences in objects otu.table, seqtab.export, seqtab.nochim.

\# Adjust sample names to match with metadata

colnames(dada res\$otu.table) = tools: :file path sans ext(colnames(dada res\$otu.table))

Taxonomic assignment. assign taxo fun function uses IDTAXA function from DECIPHER package, and allows to use two different databases. It keeps the best assignment on two criteria, resolution (depth in taxonomy assignment) and confidence (value givenby IDTAXA). The final taxonomy is validated by checking for multiple ancestors (i.e. same species assigned to different genus) and incongruities (i.e. empty fields or incomplete lineage) correction step.

We share the latest databases we use in the IDTAXA format in this link. Users can also generate your own IDTAXA formatted database following those instructions and scripts we provide at this page.

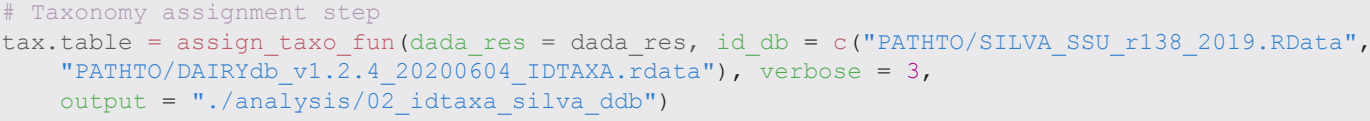

Main file outputs:

- robjects.Rdata with taxonomy in phyloseq format in tax. table object.

- final_tax_table.cSv the final assignation table (tax.table) outputed in CSV format.

- allDB tax table.CSV raw assignations from the two databases, mainly for debugging.

Table 1. Read tracking table overview.

\begin{tabular}{|l|l|l|l|l|}
\hline sample.id & input & filtered & denoisedF & nonchim \\
\hline SRR6365127.fastq & 35690 & 33511 & 33402 & 33402 \\
\hline SRR6365128.fastq & 38871 & 38183 & 38111 & 38046 \\
\hline SRR6365129.fastq & 50970 & 49765 & 49631 & 49092 \\
\hline SRR6365130.fastq & 44207 & 42657 & 42524 & 42524 \\
\hline SRR6365131.fastq & 68008 & 66517 & 66257 & 66087 \\
\hline SRR6365132.fastq & 44014 & 42158 & 41945 & 41848 \\
\hline SRR6365133.fastq & 36704 & 35884 & 35799 & 35444 \\
\hline ... & $\ldots$ & $\ldots$ & $\ldots$ & $\ldots$ \\
\hline
\end{tabular}


Phylogenetic tree. The phylogenetic tree from the representative sequences is generated using phangorn and DECIPHER packages.

tree $=$ generate_tree_fun(dada_res)

Phyloseq object. To create a phyloseq object, we need to merge four objects and one file:

- the ASV table otu. table and the representative sequences seqtab. nochim in dada_res variable.

- a taxonomy table (tax. table).

- the phylogenetic tree (tree).

- metadata from from csv file.

\# Phyloseq object generation

data $=$ generate_phyloseq_fun (dada_res $=$ dada_res, tax.table $=$ tax.table, tree $=$ tree, metadata $=" . /$ sample-metadata.csv", output $=" . /$ analysis/03_phyloseq/")

Decontamination. The decontam fun function uses decontam $\mathrm{R}$ package along with control samples (PCR control) to filter out contaminants. The decontam package offers two main methods, frequency and prevalence (users can also combine those methods). For frequency method, it is mandatory to have the DNA concentration of each sample in the phyloseq object (and hence in the metadata.csv). The prevalence method does not need DNA quantification, this method allows to compare presence/absence of ASV between real samples and control samples and then identify contaminants.

Tips: sequencing plateforms often quantify the DNA before sequencing, but do not usually give the information. Just ask for it ;).

Our function integrates the basic ASV frequency $\left(F r e q=\frac{n b_{-} \text {reads }-A S V}{n b_{-} \text {total_reads }}\right)$ and minimum prevalence in overall samples filtering. We have also included an option to filter out ASV based on their taxa names for known laboratory recurrent contaminants.

Main outputs:

- robjects.Rdata with contaminant filtered phyloseq object named data.

- Exclu_out.cSv list of filtered ASVs for each filtering step.

- Krona plot before and after filtering.

- raw_asv-table.csvand relative_asv-table.csv.

- venndiag_filtering.png venn diagram showing the repartition of filtered ASVs by decontamination methods.

Here we are going to filter out ASVs representing less than $0.1 \%$ (freq) of the reads and that are present in more than 4 samples (prev). Moreover, we are excluding "unassigned" taxas for this use case. Our sample dataset do not contain control samples, this step will be skipped.

data filtered $=$ decontam fun $($ data $=$ data, domain $=$ "Bacteria", skip $=$ TRUE, number $=4000$,

freq $=0.001$, prev $=4$, unassigned $=$ TRUE, $\mathrm{krona}=$ TRUE, output $=" . /$ analysis $/ 04 \mathrm{decontam} / "$ ) 
We obtain the final phyloseq object used for downstream analysis:

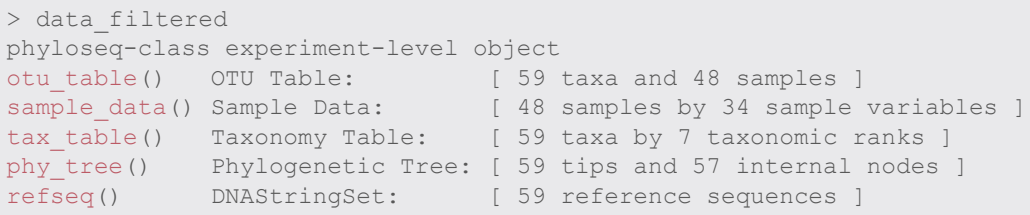

Plots, diversity and statistic analyses

Rarefaction curves. In order to observe the sampling depth of each sample we start by plotting rarefaction curves. Those plots are generated by plotly which makes them interactive. (Figure 2)

rareplot $=$ rarefaction(data_filtered, "source_location", 100)

Community composition plot. The bars fun function allows user to generate interactive community composition plot. Figure 3 presents the composition plot with relative abundances for the top 20 genera existing in our samples. The function allows to plot at different taxonomy rank and to modify the number of taxa to show.

Here two arguments controlling the composition plot aesthetics:

- Ord1 option order the sample along the $\mathrm{X}$ axis.

- Fact 1 option control labels of the $\mathrm{X}$ axis. Factl="sample.id" if user don't want the sample to be renamed.

source_location factor shows very different bacterial community between milk, cheese and cow teats environments.

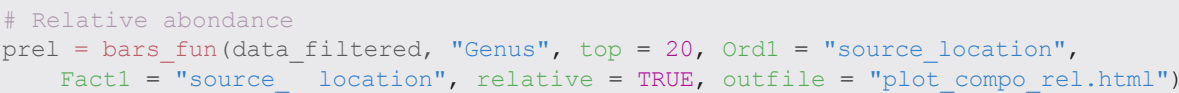

Alpha diversity. The alpha_diversity fun function can computes various alpha diversity indexes. It uses the estimate_richness function from phyloseq (Available measures : Observed, Chao1, ACE, Shannon, Simpson, InvSimpson, Fisher). Here we calculate ASV richness and Shannon index and carry out an analysis of variance on the source location factor. Sequencing depth is automatically taken into account in this test. A pairwise wilcoxon test is added to ANOVA to define which group might be significantly different

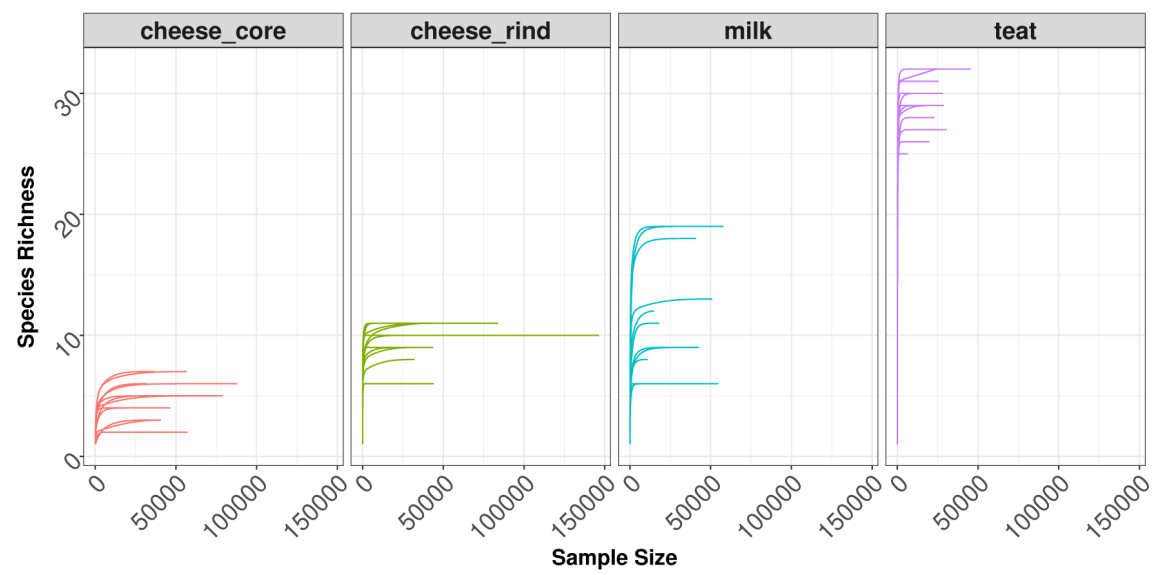

Figure 2. Rarefaction plot for all samples, separated by source_location factor. 


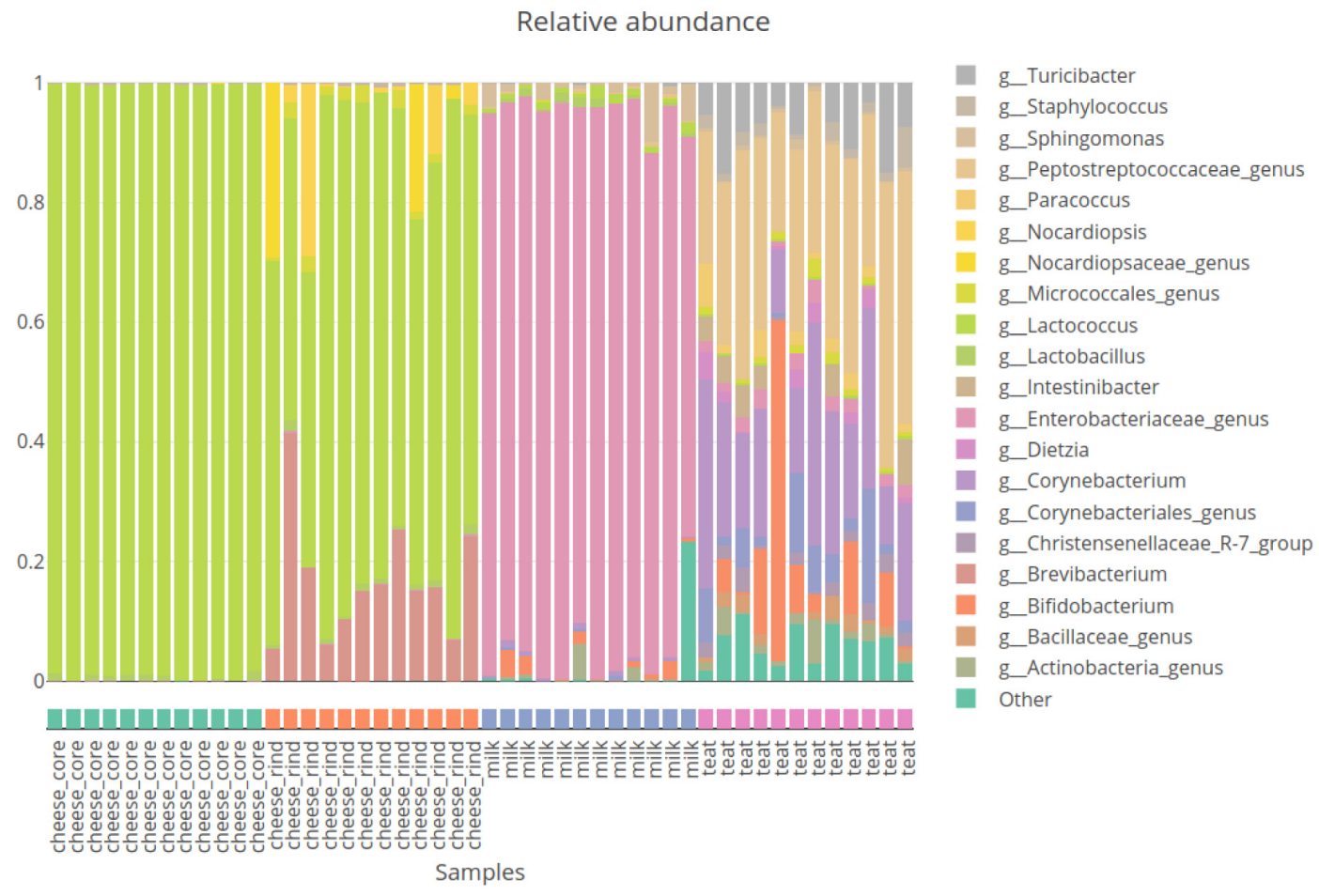

Figure 3. Composition plot of relative abundance of 20 most abundant genus.

from others. Figure 4 shows boxplots of diversity indices, cow teats environment has much more ASV than other environments. Shannon index reveals more differences between cheese rinds and cheese cores. Cheese rinds show a higher Shannon index highlighting a more balanced bacterial community.

divAlpha = diversity_alpha_fun(data = data_filtered,

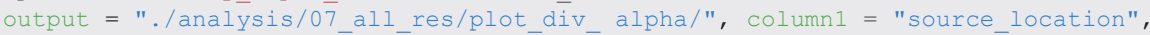

measures $=c($ "Observed", "Shannon")

Results of the analysis of variance and pairwise wilcoxon test on Shannon index:

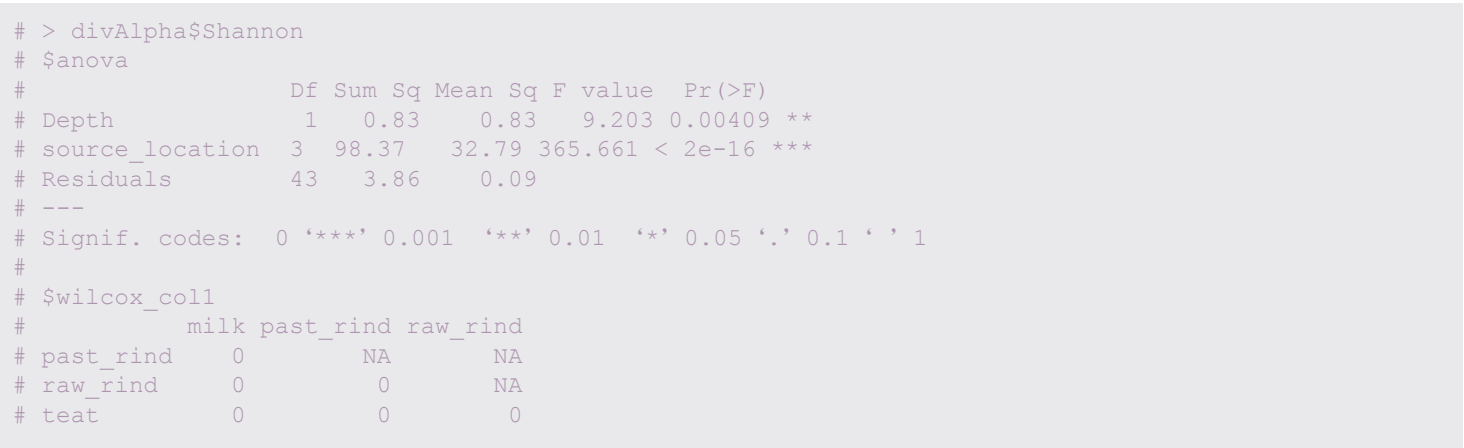

The alpha_diversity_fun function returns a list which contains:

- boxplots comparing conditions with chosen indices. (\$plot)

- a table of indices values. (\$alphatable) 


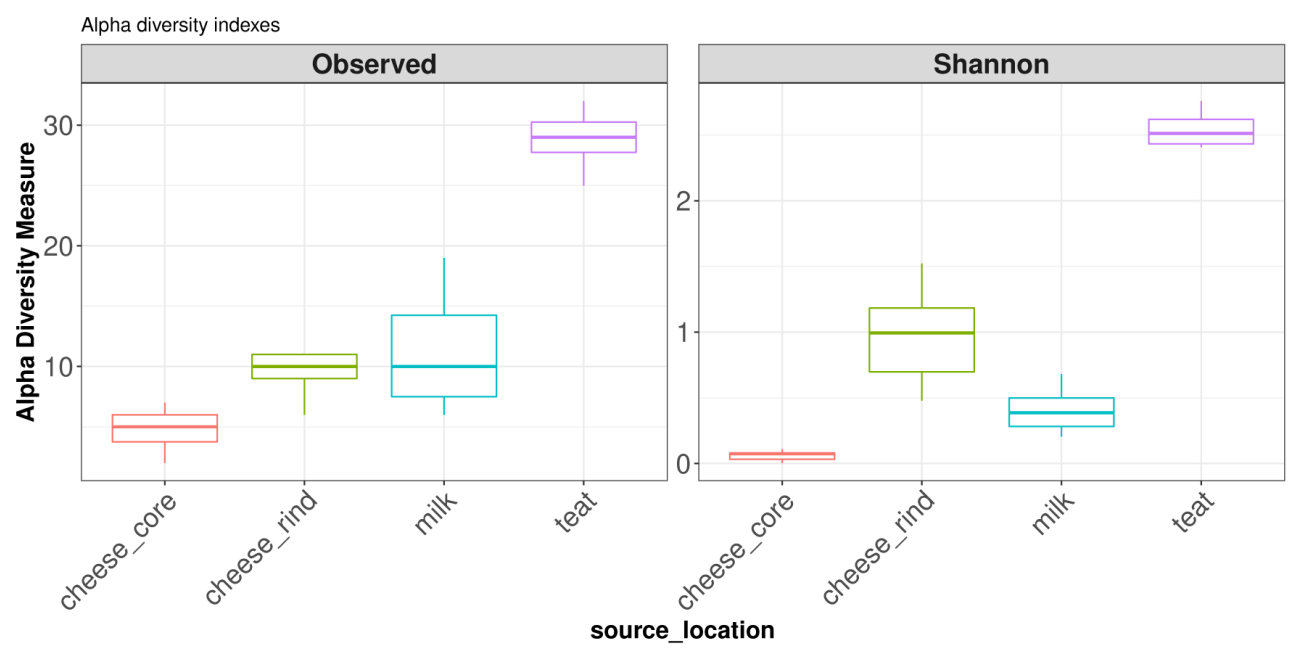

Figure 4. Boxplot of diversity alpha indexes.

And for each of the computed indices :

- an ANOVA analysis. (\$ measure $\}$ \$anova)

- a pairwise wilcox test result comparing conditions and giving the pvalue of each comparison tested: \$measure\}\$wilcox_col1: wilcox test results on the first or unique factor, \$\{measure \$wilcox_col2_fdr: wilcox test results on the second factor, \$\{measure\}\$wilcox_col2 collapsed: wilcox test results on collapsed factor 1 and factor 2 .

- a mixture model if your dataset includes repeated measures, ie. column3 option. (\$ $\{$ measure $\}$ anovarepeat, \$ $\{$ measure $\}$ \$mixedeffect)

Beta diversity. The diversity_beta_light function allows to generate specific tests and figures ready to publish in rmarkdown report as in the example below. It is based on the vegan package function vegdist for the distance calculation and phyloseq-extended in addition to ordinate funtion for the ordination plot.

We include statistical tests to ease the interpretation of results. A permutational ANOVA is carried out on matrix distance to compare groups by testing if centroids and dispersion are equivalent for all groups. User have to inform $\mathrm{COl}$ argument and optionally $\mathrm{COV}$ (covariable) to assess PERMANOVA to determine significant differences between groups. A pairwise-PERMANOVA is processed to determine which condition is significantly different from another (based on p-value).

As a return, you will get a list that contains:

- An ordination plot (\$plot).

- The permANOVA results (\$permanova).

- The pairwise permANOVA (\$pairwisepermanova)

Here we present results of beta diversity analysis on source_location factor, Figure 5 show ordination plot and it confirms the big differences between community of cheese, milk and cow teats.

divBeta $=$ diversity beta light $($ psobj = data filtered,

output $="$./analysis/07_all_res/plot_div_beta/", col = "source_location", cov = NULL,

disto = "bray", ordo = "MDS")

The permanova tests on BrayCurtis distance shows significant p-value for the source_location factor. Pairwise permanova test is used to define which level of the factor tested is significantly different from others. 


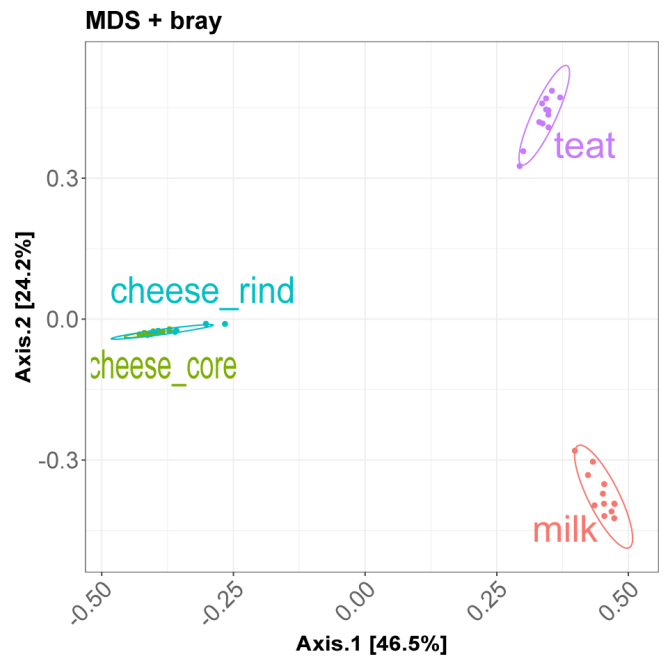

Figure 5. Ordination plot: MDS on Braycurtis distance.

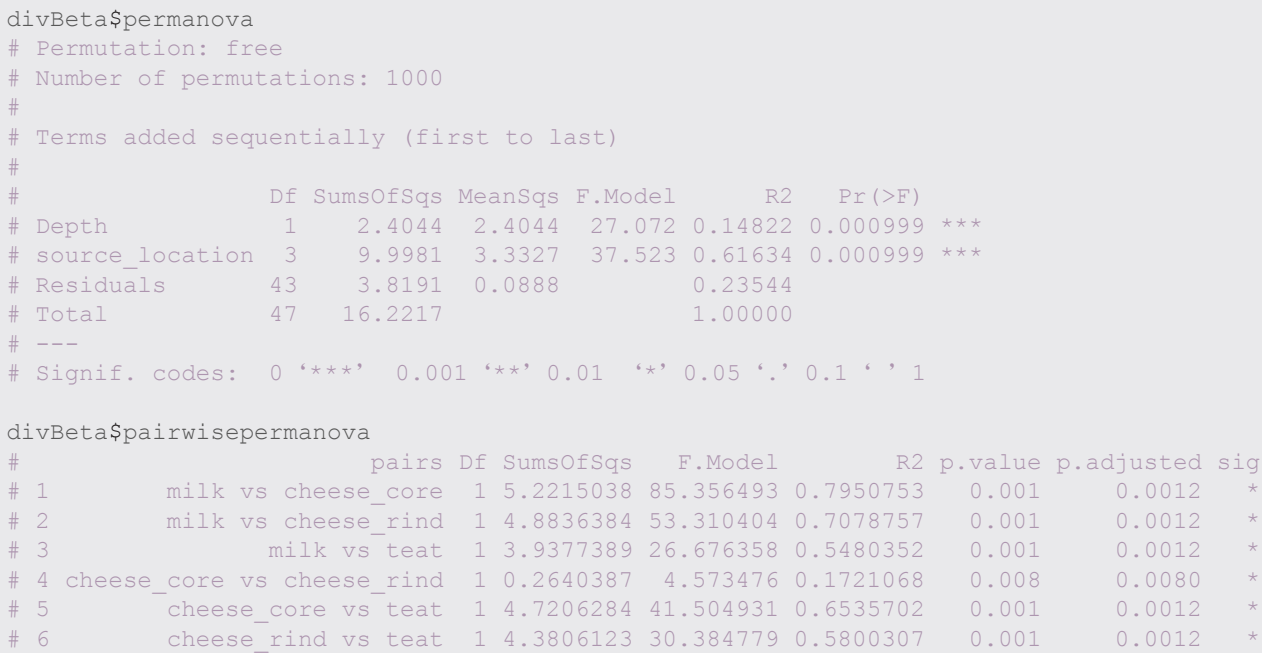

Differential analysis. We choose three different methods to process differential analysis which is a key step of the workflow. The main advantage of the use of multiple methods is to cross validate deferentially abundant taxa between tested conditions. For this use case, we choose to focus on milk and cow teat environment to compare community at genus level.

Metacoder Metacoder is the most simple differential analysis tool of the three. Counts are normalized by total sum scaling to minimize the sample sequencing depth effect and it uses a Kruskal-Wallis test to determine significant differences between groups. The metacoder fun function allows the user to choose the taxonomic rank, which factor to the test (column1), and a specific pairwise comparison (comp) to launch the differential analysis.

It produces pretty graphical trees, representing taxas present in both groups and coloring branches depending on which group this taxa is more abundant (Figure 6). Two trees are produced, a raw one, where everything is displayed and a filtered one where only significant features are represented ( $\mathrm{p}$-value $<=0.05$ ). 


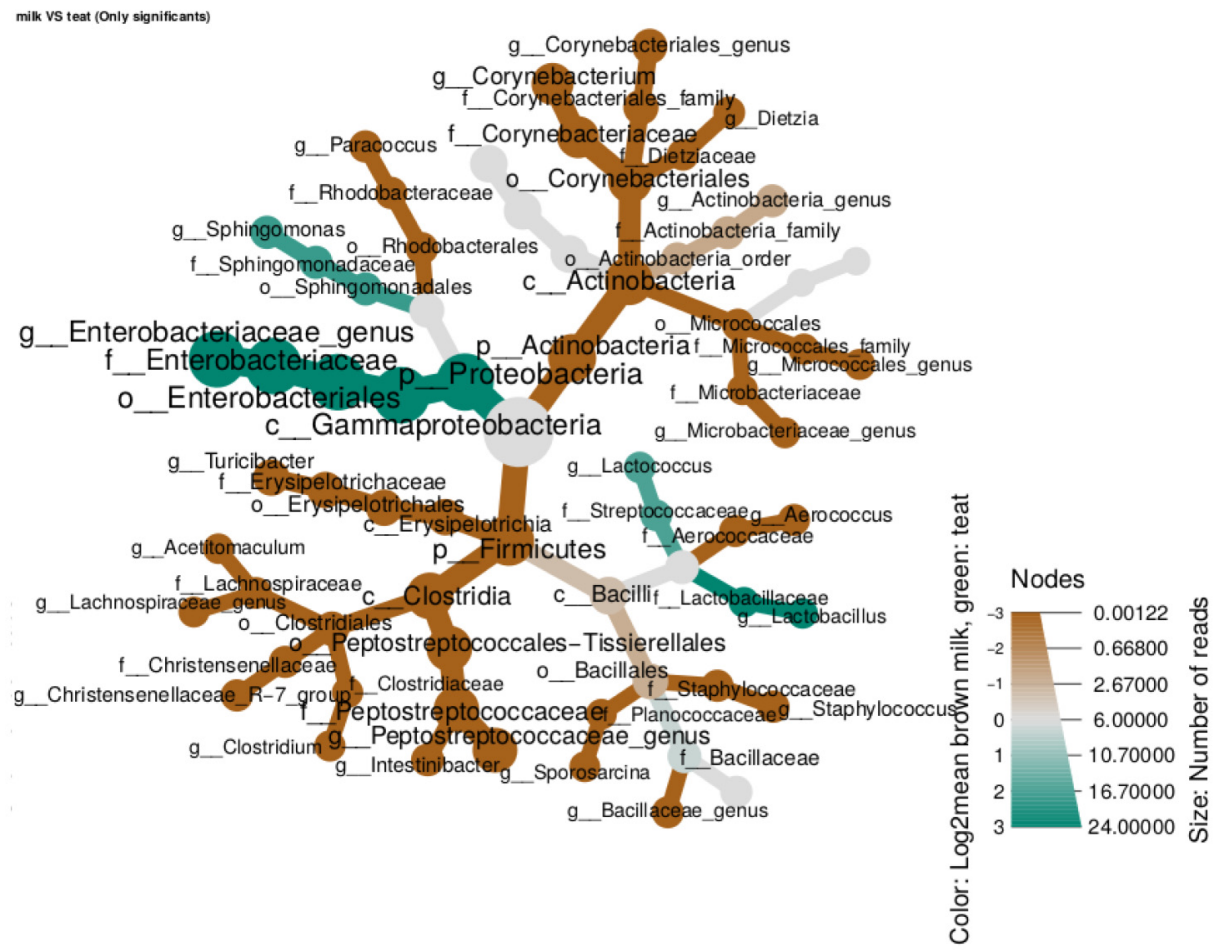

Figure 6. Heattree of significant features generated by metacoder package.

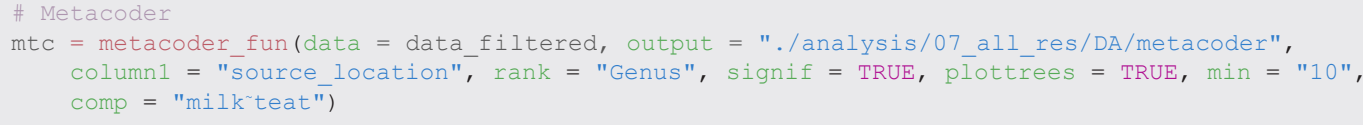

Main output is a list with :

- for each comparison ( $\{$ comparison $\}$ ), two heattree, one with all features (\$ $\{$ comparison $\}$ raw) and an other one with only significant features (\$ comparison\} \$signif).

- a table with all wilcoxon test results (\$table).

DESeq2 DESeq2 is a widely used method, primarily for RNAseq applications, for assessing differentially expressed genes between controlled conditions. Its use for metabarcoding datas is sensibly the same and well documented. The deseq2 fun allows to process differential analysis as metacoder fun, and users can choose the taxonomic rank, the factor to test and which condition to compare. DESeq 2 algorithm uses negative binomial generalized linear models with VST normalization (Variance Stabilizing Transformation).

Main output is a list with:

- a plot showing Log2FoldChange value of each significant feature (\$ comparison $\}$ plot).

- a table with statistics (LogFoldChange, pvalue, adjusted pvalue...) (\$ comparison $\}$ table).

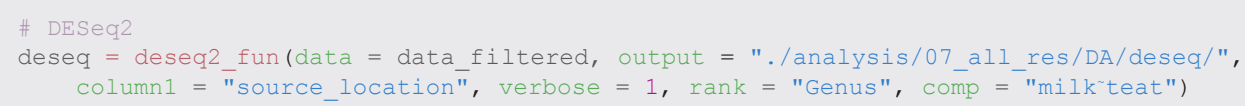

MetagenomeSeq MetagenomeSeq uses a normalization method able to control for biases in measurements across taxonomic features and a mixture model that implements a zero-inflated Gaussian distribution to account for varying depths of coverage. As deseq2 fun, metagenomeseq fun returns a table with statistics and a plot with significant features for each comparison. 
\# Metagenomeseq

mgseq $=$ metagenomeseq_fun $($ data $=$ data_filtered, output $=$ "./analysis/07_all_res $/$ DA $/ \mathrm{metagenomeseq} / "$, column 1 = "source location", verbose $=1$, rank = "Genus", comp = "milk teat")

Results aggregation step The aggregate fun function allows to merge the results from the three differential analyses methods computed previously to obtain one unique table with all informations of significant differentially abundant features. Figure 7 shows the most significant and differential abundant Genera between the two environments.

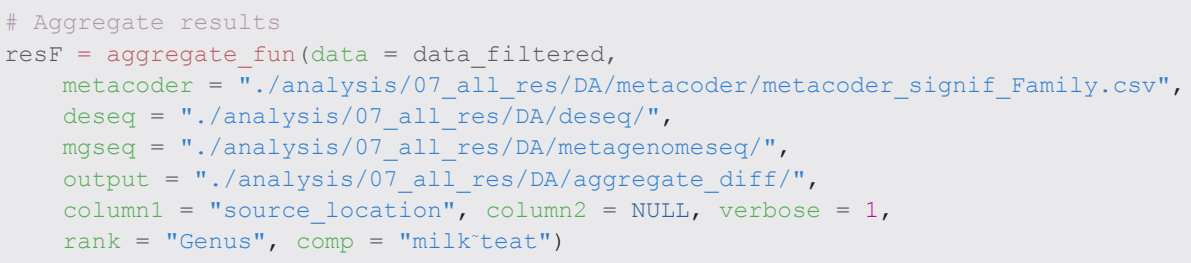

The generated table include the following fields:

- seqid: ASV ID.

- Comparaison: Tested comparison.

- Deseq / metagenomeSeq / metacoder: differentially abundant with this method (0 no or 1 yes).

- sumMethods: sum of methods in which feature is significant.

- DESeqLFC: Log Fold Change value as calculated in DESeq2.

- absDESeqLFC: absolute value of Log Fold Change value as calculated in DESeq2.

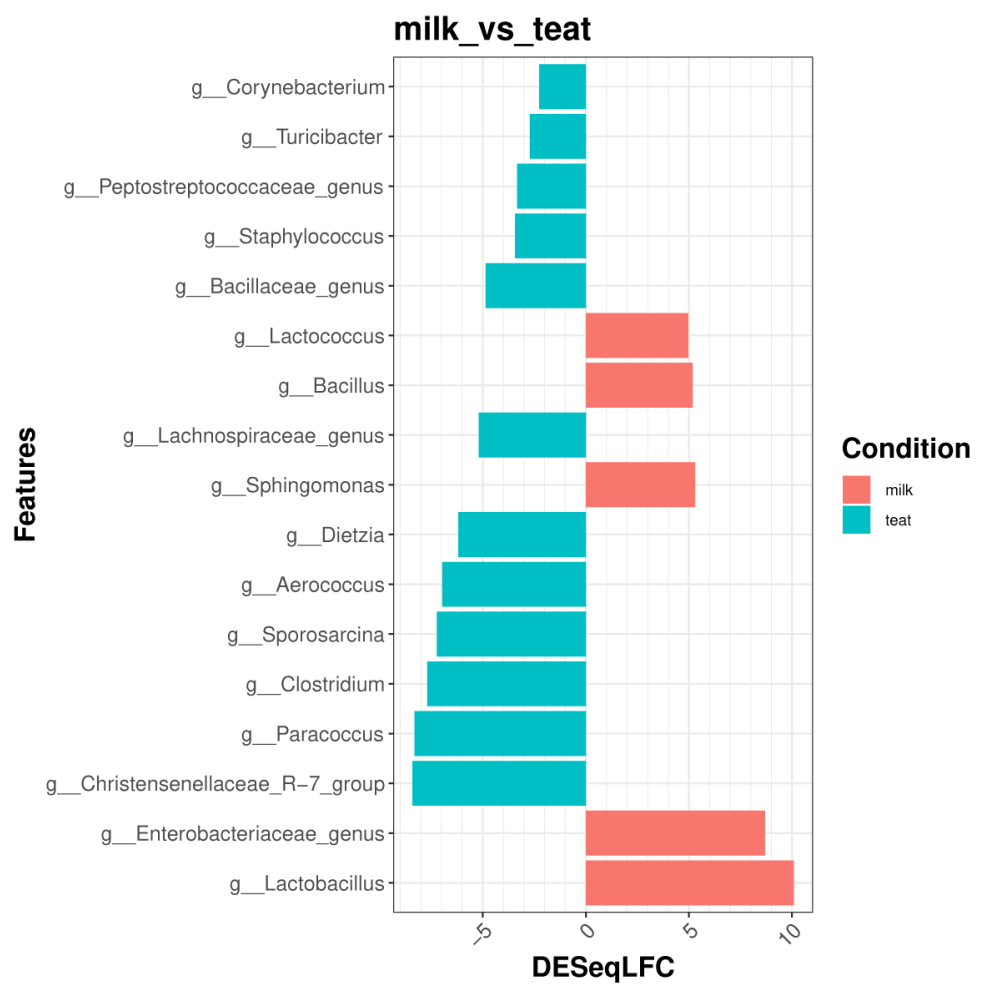

Figure 7. Top significant and differentially abundant genera between Milk and Teat samples. 
- MeanRelAbcond1 / MeanRelAbcond2: Means relative abundance in condition 1 and 2.

- Condition: in which the mean feature relative abundance is higher.

- Taxonomy and representative sequence.

Here is an overview of the aggregate_fun table informing in which methods each feature is significant, their DESeq2 LogFoldChange value, taxonomy and representative sequences:

\begin{tabular}{|c|c|c|c|c|c|c|c|c|c|}
\hline \# 1 & a $0 b c 0 e e 9 c 9$ & milk_vs_te & 1 & 1 & & 1 & 3 & 4.9664456 & 4.9664456 \\
\hline \# 2 & $13 \mathrm{~b} 622 \mathrm{bf} 4 \mathrm{~d}$ & milk_vs_te & 1 & 1 & & 1 & 3 & 8.6935035 & 8.6935035 \\
\hline 4 & d47e07353a & milk_vs_te & 1 & $c$ & & 1 & 2 & -2.2647154 & 2.2647154 \\
\hline \# 5 & $7 f 32 \mathrm{bad} 90 \mathrm{a}$ & milk_vs_te & 1 & c & & 1 & 2 & -2.7186798 & 2.7186798 \\
\hline 6 & ae 592 ee 947 & milk_vs_te & 1 & 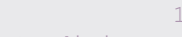 & & 1 & 3 & 5.2998345 & 5.2998345 \\
\hline 2 & 9.03565 & 2.19 & $93079 e-$ & milk k & Bacteri & $\ldots$ & Ente & erob ATATTGC & CACA \\
\hline \# 3 & 1.31035 & $1.4 \varepsilon$ & $81454 e^{-}$ & teat $k$ & Bacteri & $\ldots$ & Pept & tost ATATTGC & $\mathrm{CACA}$ \\
\hline \# 4 & 1.51020 & 8.53 & $32074 e^{-}$ & teat $k$ & Bacteri & $\ldots$ & Cory & yneb ATATTGC & $\mathrm{CACA}$ \\
\hline$\# 5$ & 8.27220 & 7.66 & $62663 e-$ & teat $\mathrm{k}$ & Bacteri & $\ldots$ & s_Turi & icib ATCTTCG & GGCA \\
\hline 6 & 2.47775 & 5.25 & $91575 e^{-}$ & milk k & Bacteri & $\ldots$ & s_sphi & ingo ATATTGG & GACA \\
\hline
\end{tabular}

\section{Miscellaneous function}

Heatmap. User can generate an interactive heatmap with heatmap_fun function to explore relative abundance of top taxa through samples as showed in Figure 8.

\# heatmap

heatmap plot $=$ heatmap fun (data $=$ data filtered, column1 = "source location", top $=100$,

output = "./analysis/07 all res/plot heatmap/", rank = "Genus")

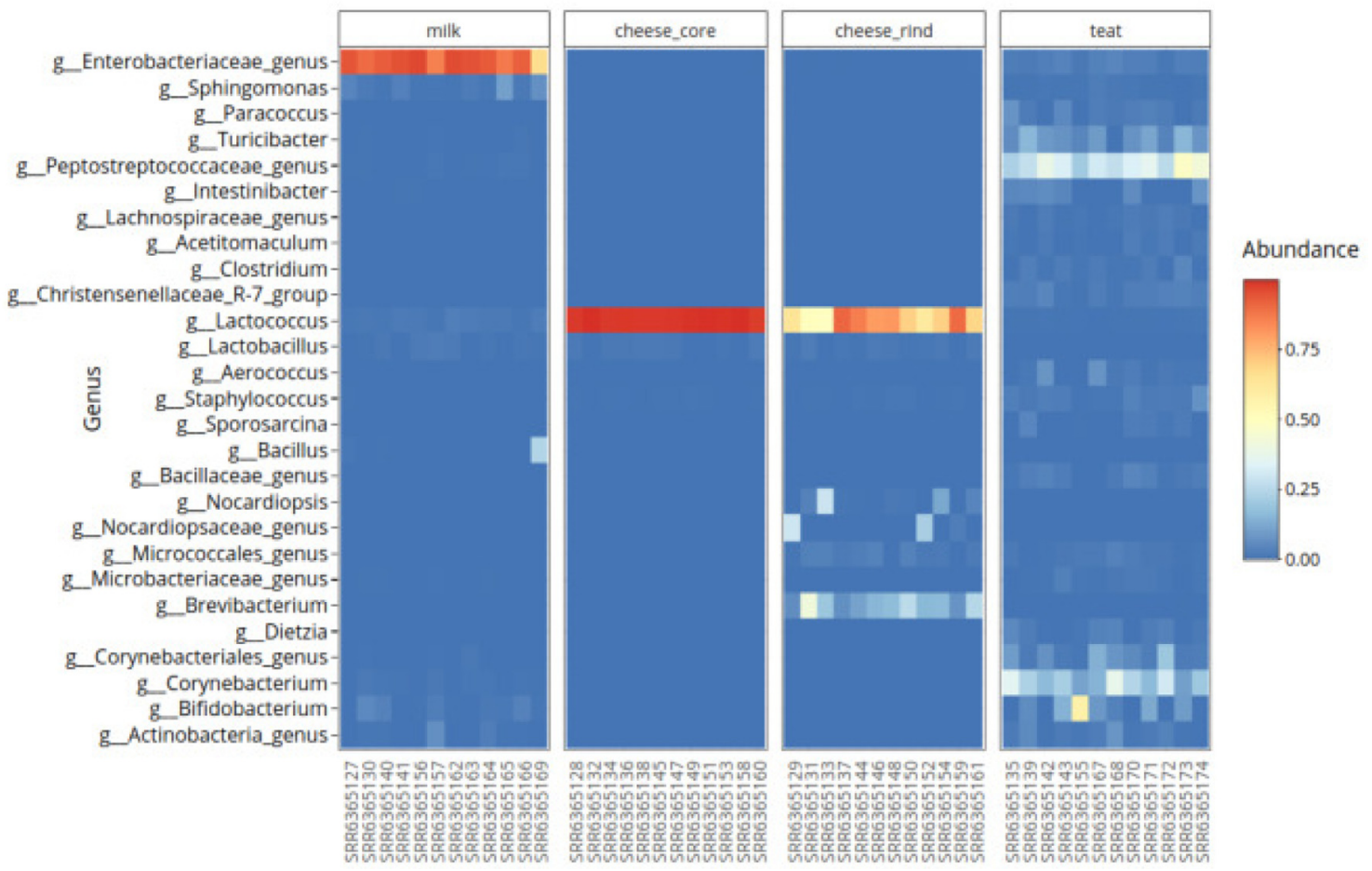

Figure 8. Heatmap of top genuses relative abundance. 
Venn diagram of shared taxa. Figure 9 shows Venn diagram comparing the three environment of this study, this method is useful to determine shared taxa between group of samples. This function uses venn: : venn function which handles up to 7 groups comparison.

\# venn

outvenn = ASVenn_fun (data $=$ data_filtered, output $=$ "./analysis/07_all_res/ASVenn/", rank = "Genus", column1 = "source location", shared = TRUE)

ASVenn_fun generate a table allowing user to find which taxa are shared between conditions.

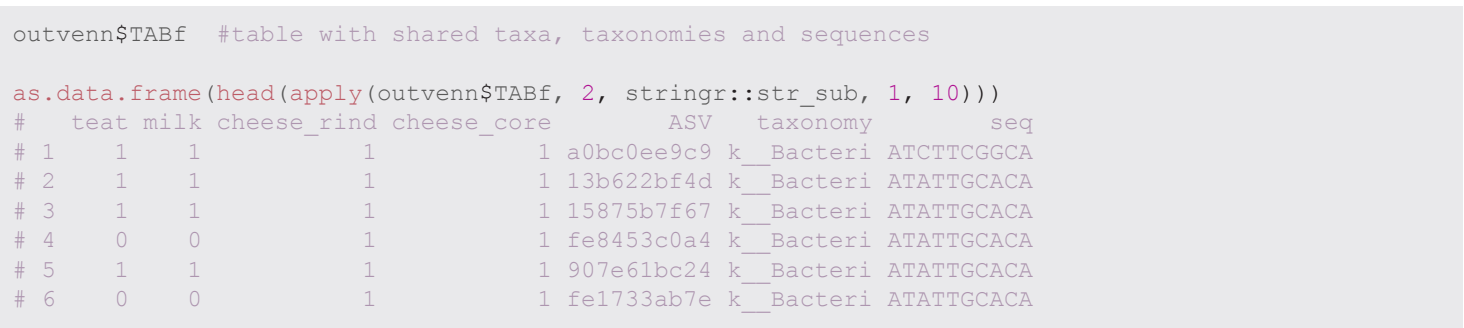

\section{Export and compatibility}

phy2cyto_fun allows to generate input files (SIF format) for Cytoscape which is useful to visualise shared taxa and easily modify each nodes and arrows position and aesthetic.

phy2tsv fun function allows user to generate tabulated format abundance table at different taxonomic rank with following commnands:

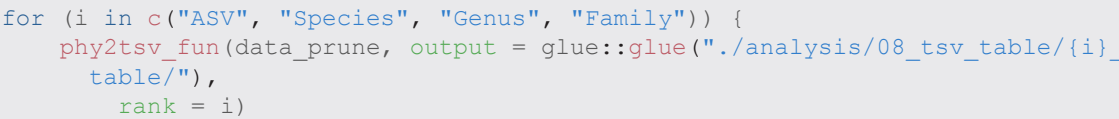

export_to_stamp_fun function creates two files that can be imported into the STAMP software.

export to stamp fun (data $=$ data, output $=" . /$ analysis/stamp/", correc $=$ TRUE)

csv2phyloseq fun function allows user to import data from other bioinformatic pipeline like FROGS, Qiime2. This function needs tabulated ASV, taxonomy, metadata and DNA sequence table as inputs. It can generate phylogenetic tree if missing and output a phyloseq object ready for downstream analyses.

\section{Conclusions}

rANOMALY allows users to handle metagenomic data from raw sequences quality control to final differential analysis with ready to publish results in an easy and reproducible manner. Users have access to all sources of

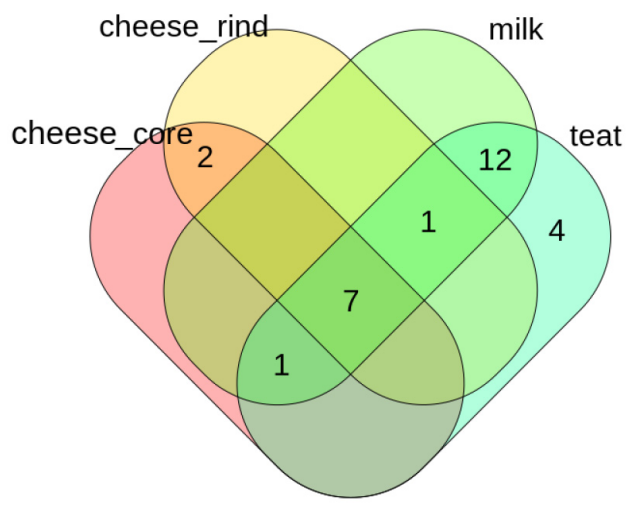

Figure 9. Venn plot of shared genuses. 
the RANOMALY package that can be deployed on any operating system or server allowing them to analyse anything from a few samples to several thousand. This workflow combines all of the latest developments in the field: the use of high resolution amplicon sequence variant, contaminant filtering, double automatic taxonomic assignation, integrated statistical analyses and four differential analyses with cross validation. rANOMALY help users those who don't have big programming skills. And since rANOMALY uses phyloseq objects and standards, original functions from the phyloseq package can still be used to split, filter and select specific samples previously to be visualized and/or tested with rANOMALY functions. For exploratory analysis and interactive experience, a shiny application based on this workflow is under active development and will be shared with the community.

\section{Software availability}

Up-to-date source code, and tutorials are available at: https://forgemia.inra.fr/umrf/ranomaly.

Package documentation is also provided at: https://umrf.pages.mia.inra.fr/ranomaly/use_case.html

Archived source code as at time of publication are available from: https://doi.org/10.5281/zenodo.433883326

License: Creative Commons Attribution 4.0 International

Here we show all packages used in this workflow and their version numbers:

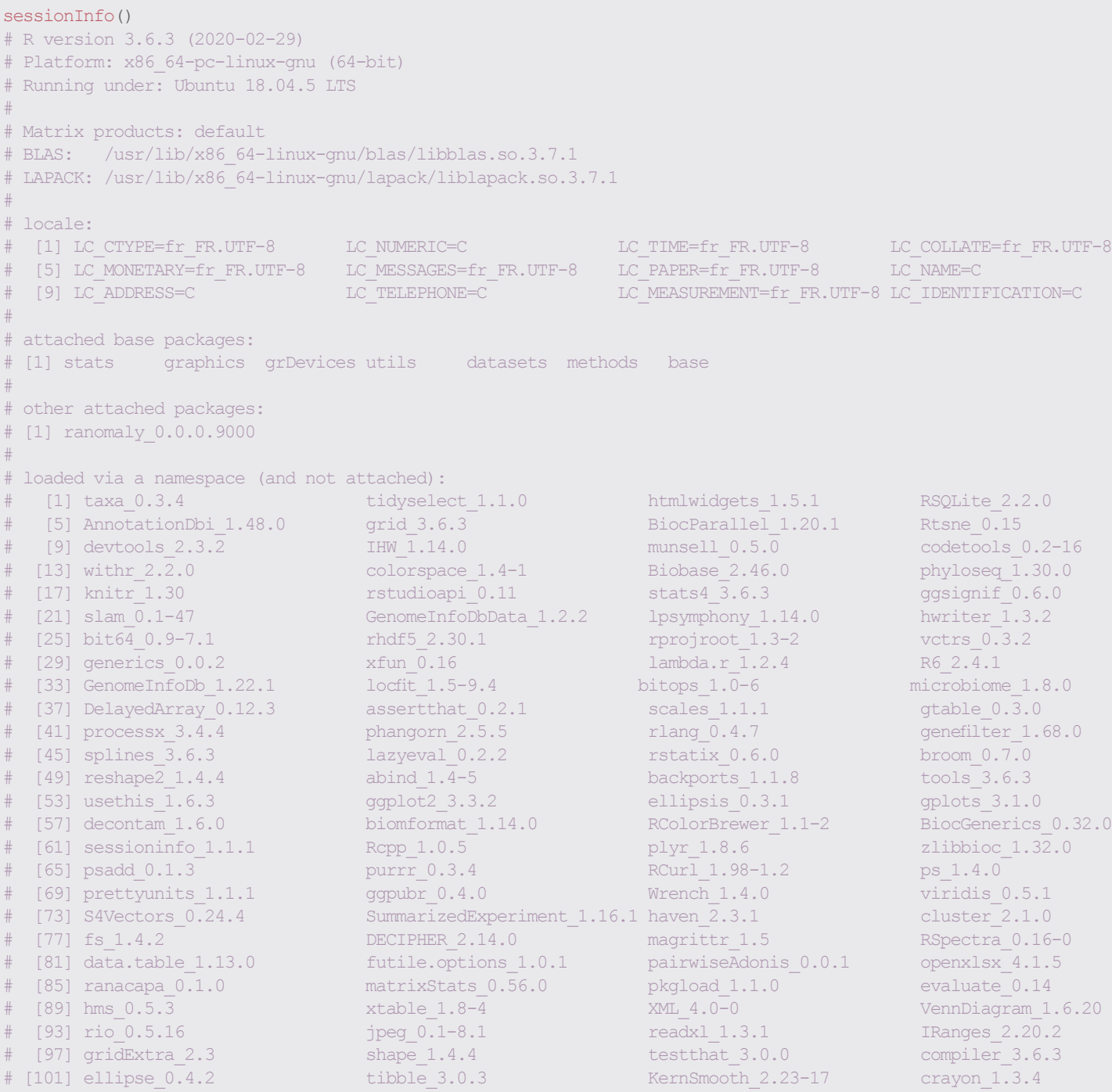




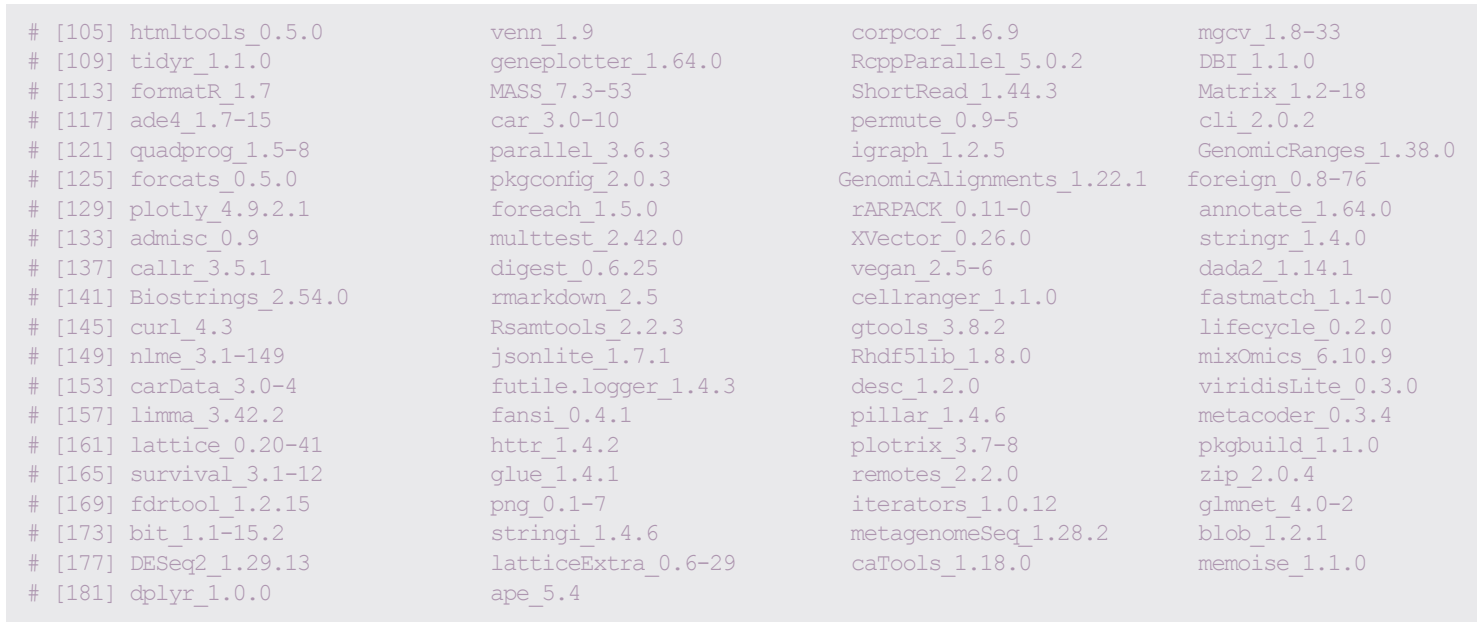

\section{Acknowledgements}

Authors deeply thank Philippe Ruiz (INRAE, MEDIS) and Marco Meola (Agroscope) for testing and feedback on the rANOMALY package.

1. Escudie F, Auer L, Bernard M, et al.: FROGS: Find, Rapidly, OTUs with Galaxy Solution. Bioinformatics. 2018; 34(8): 1287-1294. PubMed Abstract | Publisher Full Text

2. Bolyen $\mathrm{E}$, Rideout JR, Dillon MR, et al.: Reproducible, interactive, scalable and extensible microbiome data science using QIIME 2. Nat Biotechnol. 2019; 37(8): 852-857.

PubMed Abstract | Publisher Full Text | Free Full Text

3. Callahan BJ, McMurdie PJ, Rosen MJ, et al.: DADA2: Highresolution sample inference from Illumina amplicon data. Nat Methods. 2016; 13(7): 581-583.

PubMed Abstract | Publisher Full Text | Free Full Text

4. Martin M: Cut adapt removes adapter sequences from highthroughput sequencing reads. EMBnet.Journal. 2011; 17(1): 10-12.

Publisher Full Text

5. Murali A, Bhargava A, Wright ES: IDTAXA: a novel approach for accurate taxonomic classification of microbiome sequences. Microbiome. 2018; 6(1): 140 .

PubMed Abstract | Publisher Full Text | Free Full Text

6. Meola M, Rifa E, Shani N, et al.: DAIRYdb: a manually curated reference database for improved taxonomy annotation of $16 S$ rRNA gene sequences from dairy products. BMC Genomics. 2019; 20(1): 560

PubMed Abstract | Publisher Full Text | Free Full Text

7. Ritari J, Salojarvi J, Lahti L, et al.: Improved taxonomic assignment of human intestinal 16S rRNA sequences by a dedicated reference database. BMC Genomics. 2015; 16: 1056 PubMed Abstract | Publisher Full Text | Free Full Text

8. Nierychlo M, Andersen KS, Yujuan XU, et al.: MiDAS 3 : An ecosystem-specific reference database, taxonomy and knowledge platform for activated sludge and anaerobic digesters reveals species-level microbiome composition of activated sludge. Water Res. 2020; 182: 115955.

PubMed Abstract | Publisher Full Text

9. Quast C, Pruesse E, Yilmaz P, et al.: The SILVA ribosomal RNA gene database project: improved data processing and web-based tools. Nucleic Acids Res. 2013; 41(Database issue): D590-D596.

PubMed Abstract | Publisher Full Text | Free Full Text

10. DeSantis TZ, Hugenholtz P, Larsen N, et al.: Greengenes, a
Chimera-Checked 16S rRNA Gene Database and Workbench Compatible with ARB. Appl Environ Microbiol. 2006; 72(7): 5069-5072.

PubMed Abstract | Publisher Full Text | Free Full Text

11. Wang Q, Garrity GM, Tiedje JM, et al.: Naïve Bayesian Classifier for Rapid Assignment of rRNA Sequences into the New Bacterial Taxonomy. Appl Environ Microbiol. 2007; 73(16): 5261-5267.

PubMed Abstract | Publisher Full Text | Free Full Text

12. Wright ES: DECIPHER: harnessing local sequence context to improve protein multiple sequence alignment. $B M C$ Bioinformatics. 2015; 16: 322

PubMed Abstract | Publisher Full Text | Free Full Text

13. McMurdie PJ, Holmes S: phyloseq: An R Package for Reproducible Interactive Analysis and Graph-ics of Microbiome Census Data. PLoS One. 2013; 8(4): e61217. PubMed Abstract | Publisher Full Text | Free Full Text

14. Davis NM, Proctor DM, Holmes SP, et al.: Simple statistical identification and removal of contaminant sequences in marker-gene and metagenomics data. Microbiome. 2018; 6(1): 226.

PubMed Abstract | Publisher Full Text | Free Full Text

15. Sievert C: Interactive Web-Based Data Visualization with $\mathbf{R}$, plotly, and shiny. Chapman and Hall/CRC. 2020. Publisher Full Text

16. Ondov BD, Bergman NH, Phillippy AM: Krona: Interactive Metagenomic Visualization in a Web Browser. $B M C$ Bioinformatics. 2011; 12: 385

PubMed Abstract | Publisher Full Text | Free Full Text

17. Oksanen J: vegan: Community Ecology Package. R package version 2.5-5, 2019. Reference Source

18. Arbizu MP, et al.: pairwiseAdonis: Pairwise multilevel comparison using adonis. R package version 0.3. 2019.

19. Love MI, Huber W, Anders S: Moderated estimation of fold change and dispersion for RNA-seq data with DESeq2. Genome Biol. 2014; 15(12): 550. PubMed Abstract | Publisher Full Text | Free Full Text

20. Paulson JN, Stine OC, Bravo HC, et al.: Differential abundance analysis for microbial marker-gene surveys. Nat Methods. 2013, 
10(12): 1200-1202.

PubMed Abstract | Publisher Full Text | Free Full Text

21. Foster ZSL, Sharpton TJ, Grunwald NJ: Metacoder: An R package for visualization and manipulation of community taxonomic diversity data. PLoS Comput Biol. 2017; 13(2): e1005404. PubMed Abstract | Publisher Full Text | Free Full Text

22. Rohart F, Gautier B, Singh A, et al.: mixOmics: An R package for 'omics feature selection and multiple data integration. PLoS Comput Biol. 2017; 13(11): e1005752.

PubMed Abstract | Publisher Full Text | Free Full Text

23. Shannon P, Markiel A, Ozier O, et al.: Cytoscape: a software environment for integrated models of biomolecular interaction net-works. Genome Res. 2003; 13(11): 2498-2504. PubMed Abstract | Publisher Full Text | Free Full Text

24. Parks DH, Tyson GW, Hugenholtz P, et al.: STAMP: statistical analysis of taxonomic and functional profiles. Bioinformatics. 2014; 30(21): 3123-3124.

PubMed Abstract | Publisher Full Text | Free Full Text

25. Fretin M, Martin B, Rifa E, et al.: Bacterial community assembly from cow teat skin to ripened cheeses is influenced by grazing systems. Sci Rep. 2018; 8(1): 200.

PubMed Abstract | Publisher Full Text | Free Full Text

26. Theil S, Rifa E: rANOMALY. 2020.

http://wwww.doi.org/10.5281/zenodo.4338833 


\section{Open Peer Review}

\section{Current Peer Review Status:}

\section{Version 1}

Reviewer Report 22 January 2021

https://doi.org/10.5256/f1000research.30130.r76936

(C) 2021 Roux S. This is an open access peer review report distributed under the terms of the Creative Commons Attribution License, which permits unrestricted use, distribution, and reproduction in any medium, provided the original work is properly cited.

\section{Simon Roux}

Department of Energy Joint Genome Institute, Berkeley, CA, USA

This manuscript describes an R pipeline (rANOMALY) that connects together in a single simplified $\mathrm{R}$ workflow many of the standard tools now routinely used in metabarcoding data analysis. I have no doubt that such an integrated pipeline will be very useful for many researchers, especially ones not experts in bioinformatics. The modular design is also well suited for future changes and evolution of the pipeline.

The manuscript itself is very clear, nicely outlines how the different components of the pipeline are connected, and also clearly distinguish between which functions are provided by this pipeline and which functions are provided by third-party tools or packages. My only major comment is about user input (either user questions or bug reports), for which there is currently no process or web page mentioned in the manuscript. For instance, should users report bugs in the gitlab repo ( https://forgemia.inra.fr/umrf/ranomaly/-/issues)? (Incidentally, I did encounter an unexpected error with DeSeq: Error in 'assays<-'(“tmp*', value = '*vtmp*'):

please use 'assay $(x$, withDimnames=FALSE) $)<-$ value' or 'assays $(x$, withDimnames=FALSE)) <- value' when the dimnames on the supplied assay(s) are not identical to the dimnames on DESeqDataSet object ' $x$ ' In addition: Warning message: In DESeqDataSet(se, design = design, ignoreRank) : some variables in design formula are characters, converting to factors).

If the authors do intend users to submit gitlab issues, this should be specified in the conclusion and/or the software availability sections. If not, the alternative(s) path to provide feedback and report issues should be clearly described.

Other than that, I only have minor comments on the manuscript which are listed below:

Abstract:

"uses amplicon sequence variants (ASV) level for". Shouldn't this read "uses amplicon sequence variants (ASV) for"?

In the keywords, I would argue "metagenomics" should be "metabarcoding"
p. 3 Introduction: 
"Studies of microbial communities tends to" should be "Studies of microbial communities tend to"

"(FROGS ${ }^{1}$, qiime ${ }^{2}$ )": it should be made clear that these are examples of softwares but many more exist, e.g. by changing to "(e.g. FROGS ${ }^{1}$, qiime ${ }^{2}$ )", or explicitly stating "such as FROGS $^{1}$ or qiime" ${ }^{2 "}$.

"allowing to easy implementation of" should be "allowing for easy implementation of" or "allowing to easily implement".

p. 3 Raw sequence processing:

"This improves" should be clarified as "This approach improves" or "This tool improves" (to make it obvious that "this" refers to dada2).

"an additional step of cutadapt" should be "an additional step using cutadapt".

p. 4 Taxonomic assignment:

"with representing sequences" should probably be clarified as "with representative sequences for each ASV" (since the concept of "representative sequences" has not been described earlier)

"IDTAXA formatted" should be "IDTAXA-formatted"

p. 4 Phylogenetic tree:

"with AlignSeqs function from DECIPHER package" should be "with the AlignSeq function from the DECIPHER package"

"computes the likelihood of the phylogenetic tree" This may suggest that a tree exists and that this function evaluates the likelihood of this existing tree, but I believe the first function actually builds a tree? So there may be a clause missing here, e.g. "And finally, the neighbour joining function is used to build a phylogenetic tree based on the distance matrix, and the pml function computes the likelihood of the phylogenetic tree."?

\section{p. 5 Install:}

The authors may want to add a "library(devtools)" line after the install of the package devtools and before installing the package ranomaly, in case some users already have the devtools package installed.

The authors should also specify that a user will need to replace "PATHTOWORKINGDIRECTORY" with the path to their working directory on their own computer. (The same is true in the Taxonomic assignment section when users have to specify a path to the taxonomic databases).

p. 6 Processing of raw sequences:

"files name match samples name" should be "file names match sample names"

p. 6 Taxonomic assignment:

"givenby" should be "given by" 
"different genus" should be "different genera"

"correction step" should be removed, or clarified as e.g. "as part of a correction step"

"your own" should be "their own"

p. 8 Rarefaction curves:

On my version of R, I have to call "rareplot" after the line "rareplot = rarefaction(data_filtered, "source_location", 100)" otherwise no plot appears. If this is the case in most versions (which I believe it is), this line ("rareplot") should be included in the authors' instructions so that a user would not be confused to not see any plot after calling the line "rareplot = rarefaction(data_filtered, "source_location", 100)" (this is also true for the other interactive plots, e.g. community composition, alpha and beta diversity, etc).

p. 8 Community composition plot:

"source_location": there seems to be an extra space between "source_" and "location" here

p. 10 Beta diversity:

"funtion" should be "function"

"on matrix distance" should be "on the distance matrix"

"A pairwise-PERMANOVA is processed" should be "A pairwise-PERMANOVA is performed"

p. 11 Differential analysis:

"of the use of multiple" would be more clear as "of using multiple"

"cross validate" should be "cross-validate"

"which factor to the test" should be "which factor to test" or "which factor to perform the test on"

"which group this taxa is more abundant" should be "which group this taxa is more abundant in"

p. 12 DESeq2:

"and well documented": Could the authors add 1 or 2 references here?

p. 15 Venn diagram:

Shouldn't "the three environment" be "the four environments".

p. 15 Export:

"function allows user" should be "function allows users" (also for csv2phyloseq_fun).

p. 15 Conclusion:

"metagenomic data" should be "metabarcoding data" 
"help users those" should be "help users"

"big programming skills" should be "advanced programming skills"

"previously to be visualized and/or tested" should be "prior to visualization/test" or "before being visualized and/or tested"

Is the rationale for developing the new software tool clearly explained?

Yes

Is the description of the software tool technically sound?

Yes

Are sufficient details of the code, methods and analysis (if applicable) provided to allow replication of the software development and its use by others?

Yes

Is sufficient information provided to allow interpretation of the expected output datasets and any results generated using the tool?

Yes

Are the conclusions about the tool and its performance adequately supported by the findings presented in the article?

Yes

Competing Interests: No competing interests were disclosed.

Reviewer Expertise: metagenomics, microbial ecology, phage genomics, viral ecology

I confirm that I have read this submission and believe that I have an appropriate level of expertise to confirm that it is of an acceptable scientific standard.

Reviewer Report 12 January 2021

\section{https://doi.org/10.5256/f1000research.30130.r76934}

(C) 2021 Murat F. This is an open access peer review report distributed under the terms of the Creative Commons Attribution License, which permits unrestricted use, distribution, and reproduction in any medium, provided the original work is properly cited.

\section{Florent Murat}

Heidelberg University, Heidelberg, Germany

Theil and Rifa developed here the R package rANOMALY for data analysis of $16 \mathrm{~S}$ and ITS amplicons based sequencing. They provide a workflow and a toolkit that are very useful for bioinformaticians as well as for beginners who want to perform complete marker gene sequencing data analyses 
from fastq treatment (contaminant filtering, taxonomic assignment, alpha and beta diversity indexes) to statistical analysis (using three main differential analysis methods, DESeq2, Metacoder, metagenomeSeq) in an automatic, step by step, and reproducible way. Thus, this is a suitable workflow for exploratory and educational purposes. I was pleased to be able to reproduce all analyses and figures following the use case of the paper.

Hence, I only have a few minor issues that should be discussed:

1. The authors mentioned that the developed package is customizable. This is an important aspect given that projects are different from one to the other, and bioinformatic tools evolve tremendously fast so it will be crucial to adapt/update the workflow accordingly. The workflow actually allows this, however, it would be useful (particularly for beginners) to provide a vignette/tutorial on the gitlab page (and/or in a section of the paper) showing how this can be done with simple examples. The use case is based on Illumina sequences, a use case dealing with Ion torrent sequences could be relevant in this context.

2. The use case is based on a dataset from Fretin et al. 2018, it would be relevant to interpret the results of the workflow, and to describe to what extent they match or complement the results of the previous analysis. This would be relevant to benchmark the workflow and highlight the novelties brought by this analysis.

3. The access to the use case fastq sequences and to the IDTAXA formatted databases is given via links in the text. It would be convenient for users working with remote servers to download it with command lines. Please provide command lines to do so (via wget for instance), either in the text or on the gitlab page.

4. There is a typo on page 6 , the IDTAXA formatted database that can be downloaded from the link is DAIRYdb_v1.2.4_20200603_IDTAXA.rdata and not DAIRYdb_v1.2.4_20200604_IDTAXA.rdata.

5. Page 8: In the "bars_fun" function, additional space has been introduced between "source_" and "location" leading to an error if one runs this piece of code.

6. Page 9: In the "diversity_alpha_fun" function, the font has been modified for the closing double quotes after "Observed" and "Shannon". Thus, this leads to an error if one runs this piece of code.

7. Page 15: In the "phy2tsv" function, the phyloseq object "data_prune" was never created upstream. Would it be better to test the function "phy2tsv" with the phyloseq object "data" previously created in the use case?

Is the rationale for developing the new software tool clearly explained? Yes

Is the description of the software tool technically sound? Yes

Are sufficient details of the code, methods and analysis (if applicable) provided to allow replication of the software development and its use by others? 
Yes

Is sufficient information provided to allow interpretation of the expected output datasets and any results generated using the tool?

Yes

Are the conclusions about the tool and its performance adequately supported by the findings presented in the article?

Yes

Competing Interests: No competing interests were disclosed.

Reviewer Expertise: Genomics, Bioinformatics, Evolution

I confirm that I have read this submission and believe that I have an appropriate level of expertise to confirm that it is of an acceptable scientific standard.

The benefits of publishing with F1000Research:

- Your article is published within days, with no editorial bias

- You can publish traditional articles, null/negative results, case reports, data notes and more

- The peer review process is transparent and collaborative

- Your article is indexed in PubMed after passing peer review

- Dedicated customer support at every stage

For pre-submission enquiries, contact research@f1000.com 